\title{
28.8.2014: Ein Paradebeispiel für die erfolgreiche Einführung der Quote in der Personalführung ..., ein spannender Abend im Netzwerk des djb beim Rundfunk Berlin-Brandenburg rbb
}

\author{
Kathrin Otto, \\ Vorstand djb-Landesverband Berlin
}

Der rbb hat die höchste Frauenquote in Führungspositionen in der ARD (ca. 50\%). Die Intendantin Frau Dagmar Reim, der rbb-Chefredakteur, Herr Singelnstein, sowie die Abteilungsleiterin Personalstrategie und -entwicklung, Frau Dr. Heser, haben für uns im djb-Landesverband Berlin vorgetragen, was der rbb seit Jahren in der Personalstrategie plant und in der Personalentwicklung erfolgreich umsetzt. Hierbei gilt der Grundsatz: „Wo, wann und wie ich arbeite, sollte verhandelbar sein."

Ein persönlicher Bericht:

Donnerstagabend, 28. August 2014, kurz vor 19.00 Uhr, die monatliche Veranstaltung der djb-Regionalgruppe Berlin findet dieses Mal an einem außergewöhnlichen Ort statt: Im 14. Stock des rbb-Standortes Berlin, Masurenallee.

Im Fahrstuhl treffe ich andere Teilnehmerinnen auf dem Weg nach oben. Wir freuen uns schon auf den Blick über Berlin, der muss in der Dämmerung phantastisch sein.

Oben angekommen, erwartet uns ein Büffet; dies, so spricht sich schnell herum, ist auf die Initiative von derjenigen zurückzuführen, auf deren Einladung wir heute Abend hier sein dürfen, der Intendantin des rbb, Frau Reim. Sie wisse aus Erfahrung, so heißt es, dass man (und frau) um diese Uhrzeit nach der Arbeit hungrig sei. Stimmt, wir greifen gern zu.

Schon bald bestückt sich das Podium, auf dem neben Frau Reim auch der Chefredakteur Herr Singelnstein sowie die Leiterin der Abteilung Personalstrategie und -entwicklung, Frau Dr. Heser, Platz nehmen. Kurz danach geht das inhaltliche Feuerwerk los. Frau Reim leitet ein, und gleich ihr erster Ansatz spiegelt eine Erfahrung von mancher Zuhörerin wider: Wandel in einer hierarchischen Organisation müsse von oben kommen, der Versuch eines Wandels von unten sei schwierig bis unmöglich.

Auch sei sie, so Frau Reim, inzwischen eine Anhängerin der Einführung einer Frauenquote für Führungspositionen. Schon unter Bundeskanzler Schröder seien freiwillige Quoten mit der Wirtschaft vereinbart worden - ohne nennenswerten Erfolg. Aber, auch das führt sie an, der Erfolg einer Quote sei eine Frage der Unternehmenskultur. Wir horchen auf, was meint sie damit?

Frau Reim schildert ein Beispiel aus ihrer unmittelbaren beruflichen Umgebung: Als sie beschlossen habe, die Leitung ihrer Intendanz zwei Halbtagskräften anzuvertrauen, habe sie mit allen Direktoren und der Direktorin persönlich darüber ge- sprochen, für die Entscheidung geworben und sie gegen manche Kritik verteidigt. Und es sei ein Erfolg geworden.

Was war passiert? Frau Reim nennt als entscheidenden Faktor etwas, das sie mit Binnen-Klima bezeichnet: Kein Mitarbeitender darf alle Vergünstigungen bekommen, es müsse immer einen Ausgleich zwischen allen Interessen geben, jede und jeder müsse Rücksicht auf die Kolleginnen und Kollegen und deren Belange nehmen. Unterstützung müsse auch außerhalb der vereinbarten Zeiten angeboten werden, wenn dienstliche Nöte es gebieten.

Denn über allem stünde eins: Das Programm des rbb muss gesendet werden, mit höchstmöglichem Standard, egal ob live oder Aufzeichnung, egal ob Radio oder Fernsehen. Beim Erreichen dieses Ziels sei Flexibilisierung jedoch immer möglich.

Herr Singelnstein bestätigt an dieser Stelle Synergieeffekte: Die Praxis zeige, dass man bei zwei Teilzeitkräften à 50 Prozent mehr als hundertprozentige Arbeitsleistung bekäme. Die Teilzeitkräfte zeigten im Schnitt ausgeglichenere Leistungen und weniger Müdigkeitsphasen.

Und Frau Dr. Heser schließt gleich an, dass der Erfolg des Job-Sharing-Modells bei der Leitung der Intendanz schnell Vorbild für andere Bereiche geworden sei, auch für ihren; auch sie teile sich die Leitung der Abteilung.

Und diese und andere Aktionen seien auch nötig, um Beschäftigte für den rbb zu gewinnen. Tarifliche Regelungen begrenzen die Möglichkeiten finanzieller Anreize, doch die Entwicklung der Demographie in Deutschland fordere auch den rbb heraus. Und Frau Dr. Heser erläutert die weiteren Anreize, die im rbb angewandt werden:

Eine gezielte Frauenförderung trägt dazu bei, qualifizierte Beschäftigte zu gewinnen und an den rbb zu binden. Bedarfsgerechte Fortbildungen und spezifische Seminarangebote helfen ebenfalls. Vor allem für die technischen Berufe laufen spezifische Nachwuchsprojekte wie „Frauen und Technik“. Auch gibt es die Möglichkeit eines dualen Studiums, das sehr gerne von Frauen aufgenommen wird. Der rbb ist Partner des „Nationalen Paktes für Frauen in den MINT-Berufen “ und wirbt durch die Teilnahme am Girls’ Day für sich. Offensichtlich mit Erfolg: Bei den Führungskräften hat der rbb die höchste Frauenquote innerhalb der ARD.

Doch nicht nur das, auch Maßnahmen zur Flexibilisierung der Arbeitszeit werden groß geschrieben:

So gibt es diverse Arbeitszeitmodelle, insgesamt 230 verschiedene, von der tageweisen Freizeit über regelmäßige Freizeit an Vor- und/oder an Nachmittagen bis zu Blockmodellen. Immer 
individuell angepasst an die persönlichen und die dienstlichen Notwendigkeiten: „Die Sendung muss gesendet werden.“

Auf Arbeitszeitreduzierungen müssen sich die Beschäftigten nur befristet festlegen, um auf mögliche Änderungen in der Lebensphase reagieren zu können. Wo immer möglich, gibt es Vertrauensarbeitszeit. Wir horchen auf, das Binnen-Klima scheint zu wirken.

Frau Reim verhehlt nicht, dass für die Gestaltung von Arbeitszeiten ein hoher Koordinationsaufwand nötig ist. Gefährden gewünschte freie Zeiten eine Sendung, ist die für die Zeitkoordination zuständige Person herausgefordert. Aber die Ergebnisse rechtfertigten den Aufwand.

Und eine zweite Schwierigkeit verhehlt sie nicht, befristete Arbeitszeitreduzierungen von Stammkräften führen zu befristeten Arbeitsverträgen für Vertretungskräfte, dies sei nicht zu ändern.

Doch der rbb geht weiter, verschiedene Varianten der Kinderbetreuung stehen im Angebot, wie das Vorhalten von Belegplätzen in Kitas und von Eltern-Kind-Zimmern in Berlin und Potsdam, ein Kinderferienprogramm, das die ganztägige Betreuung von Kindern während der sechswöchigen Sommerferien und Kita-Schließzeiten sicherstellt. Unter Leitung einer ausgebildeten Pädagogin und unter Einbezug ehemaliger Mitarbeitender, bei deren Engagement manchmal auch ein wenig, oder mehr, Überredungskunst anzuwenden sei, wie Frau Reim attestiert.
Insgesamt alles mit Erfolg, wichtig für den rbb sei aber, so betont Frau Dr. Heser, die Erkenntnis, dass Vereinbarkeit von Familie und Beruf auch beinhalte, dass die Pflege Angehöriger unterstützt würde. Hier würden die Aktivitäten gerade ausgeweitet. Ebenso wie bei der Einführung der Telearbeit, die vom Arbeitsort unabhängiger mache.

Natürlich gab es nach der Podiumsrunde viel zu diskutieren, erst im gesamten Plenum, dann bilateral beim zweiten Gang zum Buffet. Da waren noch weitere praktische Erfahrungen mit dem Prinzip der geteilten Führungspositionen zu erfahren: Mitarbeitende bevorzugen oft klare Zuständigkeiten, bewährt habe sich deren Aufteilung unter den beiden Führungskräften eines Bereiches unter Berücksichtigung von deren Verfügbarkeit. Für schnelle Mitzeichnungen sollte die Person zuständig sein, die auch ad hoc zur Verfügung steht.

Zu erfahren war auch, dass für diese Art der Führung spezifische Qualitäten der Führungskraft gefragt seien. Delegationsfähigkeit und Vertrauen in die Fähigkeiten und Loyalität der Mitarbeitenden seien entscheidend.

Leib und Geist bekamen bei diesem Einblick in die Praxis der Quote und der äußerst erfolgreichen Personalführung beim rbb viel Futter, so viel, dass mir beim Weg im Fahrstuhl nach unten auffiel, dass ich nicht ein einziges Mal aus dem Fenster geschaut hatte.

\section{Der djb gratuliert}

Mechtild Düsing

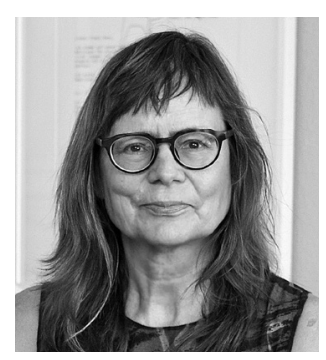

zum 70. Geburtstag. Mechtild Düsing, geb. 29. September 1944 in Bilstein Lennestadt, studierte von 1965 bis 1969 Rechtswissenschaft an den Universitäten Münster und München. Nach ihrem Referendariat beim OLG Hamm und dem Abschluss des Zweiten Staatsexamens 1973 in Düsseldorf war sie zunächst als Rechtsanwältin in einer Einzelpraxis tätig und gründete 1975 mit Kollegen die Kanzlei Meisterernst Düsing Manstetten in Münster. 1983 erfolgte ihre Ernennung zur Notarin, 1988 wurde sie zunächst Fachanwältin für Verwaltungsrecht mit Schwerpunkt Studienplatzklagen. 2006 wurde sie auch Fachanwältin für Erbrecht.

Zwischen 1990 und 2006 vertrat sie insbesondere Landwirte vor den Europäischen Gerichten in Luxemburg in Schadensersatzprozessen gegen die Europäische Gemeinschaft. Sie ist seit 2009 Vorsitzende der Arbeitsgemeinschaft Agrarrecht im Deutschen Anwaltverein (DAV). Seit 2009 ist sie Vorsitzende des Fachanwaltsausschuss Agrarrecht der Rechtsanwaltskammer Hamm, seit 2010 Fachanwältin für Agrarrecht. Sie veröffentlichte zahlreiche Publikationen und Vorträge zum
Landwirtschaftsrecht, Anwaltsrecht und zu Frauen in der Anwaltschaft.

Mechtild Düsing ist seit 2001 Mitglied des Verfassungsrechtsausschusses des DAV. Seit 2011 ist sie Mitglied des Vorstands des Deutschen Anwaltvereins (DAV). Von 2004 bis 2009 war sie Vorsitzende der Arbeitsgemeinschaft Anwältinnen im DAV und ist seitdem Vorstandsmitglied. Sie ist Genderbeauftragte des DAV-Vorstandes und Vorsitzende des Genderausschusses des DAV. Außerdem ist sie Mitglied der Arbeitsgemeinschaften Erbrecht, Internationaler Rechtsverkehr und Anwaltsnotariat im DAV.

Weiterhin ist sie Mitglied der European Women Lawyers Association (EWLA), von ZONTA International, dem Westfälischen Kunstverein Münster (Mitglied des Beirats), der Bürgerstiftung Münster (Mitglied im Kuratorium) und der AtlantikBrücke e.V. Berlin.

Mechtild Düsing ist seit 1981 Mitglied des djb und seit 1994 Vorstandsmitglied (Schatzmeisterin) der Regionalgruppe Münster. Sie engagiert sich in zahlreichen djb-Projekten, u.a. initiierte sie 2009 die Aktion „Aktionärinnen fordern Gleichberechtigung - Erhöhung des Frauenanteils in Führungspositionen deutscher Unternehmen".

Sie ist verheiratet und hat drei Kinder. 